\title{
The Use of Simulation Activities in Nursing to Increase Awareness of Patient Disabilities-Consideration of COVID-19 Practice Standards Debra Hagerty
}

Faculty of Nursing, Georgia Southern University, Armstrong Campus-Ashmore Hall 259, GA 30458, USA

\section{Introdution}

Student learning and engagement must be accomplished both in the classroom, clinical areas as well as virtually in the $21^{\text {st }}$ century. Students are supported with a variety of resources to improve metacognition, thinking and clinical learning. The global pandemic has prevented some student opportunities and prohibited attending the usual in-person patient interactions in hospitals and clinical sites that normally would be a part of the student clinical experience [1]. The variety of learning platforms driving innovation are limited only by the faculty resources and restrictions placed on student engagement to perform in the clinical and simulation environments. A simulation experience designed to introduce students to several disabilities that patients experience and are observed in the clinical area was created.

The objective was to engage students in application of equipment to simulate a variety of disabilities commonly observed in the clinical and community areas. Engagement and active student participation as learning techniques has been shown to enhance learning outcomes [2]. Active engagement supports learning. "Students learn best when learning is active, when they are mentally involved, when they engage in hands-on activities, when they are involved in a process of inquiry, discovery, investigation, and interpretation" [3]. Students engaging in hands-on activities to learn about disabilities as well as experience and sense the specific disability or sensory impairment by donning and doffing simulation equipment contributes to student active engagement and experiential learning. Opportunities such as this simulation may be used to support learning and contribute to clinical hours for students. The American Disabilities Act (ADA) of 1990 was the inspiration for this event. The definition of disability is important for students to be aware of and includes, "a physical or mental impairment that substantially limits one or more major life activities; a record or history of such an impairment; or a perception by others as having such impairment". Also important is an understanding that "a major life activity also includes the operation of a major bodily function, including but not limited to, functions of the immune system, normal cell growth, digestive, bowel, bladder, neurological, brain, respiratory, circulatory, endocrine, and reproductive functions, [4, SEC. 12101, Section 3]. The disability definitions and discussions of these patient health concerns occurred during the simulation. Currently, the education provided by nursing programs in caring for the disabled remains mostly informative, failing to engage students in active learning and transforming the information into competencies needed to effectively care for patients with disabilities [5, p. 234].

The zoom platform was used to share this experience online in real time with other students. During the global pandemic creativity in teaching actions and platform utilization support students with pedagogical diversity necessary for success. Clinical practice experiences demonstrated with simulation pedagogy provide a safe environment for student learning by using a student supported approach enabling student centered learning and learning outcomes [6]. The goal of this experience was to introduce interprofessional students to several disabilities common to clinical experiences taking theoretical knowledge and bridging to the clinical exposure in a safe simulated environment.

\section{Publication History:}

Received: April 24, 2021

Accepted: May 03, 2021

Published: May 05, 2021

\section{Keywords:}

Simulation activities, COVID-19, Patient disabilities

Students of the Institute for Healthcare Improvement (IHI) Chapter and the Omicron Delta Kappa (ODK), leadership honor society chapter hosted a collaborative interprofessional student focused event. Invitation to attend was email published to interprofessional university faculty and flyers posted around campus. The faculty advisors in attendance agreed to take attendance at the event to encourage student attendance and providing an option for credit or service credit to be applied.

\section{Orientation}

Faculties were secured on a voluntary basis. Nurses participating in the individual disability stations had minimal qualifications of a Master's of Science in Nursing. Orientation to the operational and safety considerations of the simulation equipment was thorough and took place with each individual manning a station. Question and answer sessions, disability device application and safety settings were reviewed where applicable. Manufacturer recommendations were followed. Faculties were instructed to consider and be mindful of the psychological safety of participants. Lead Faculty were available for consultation.

\section{Cleaning and Sanitizing}

Cleaning and sanitizing of the overall environment was accomplished with consideration of the Centers for Disease Control and Prevention [CDC] [7]. Products chosen were Environmental Protection Agency (EPA) approved for disinfection of surfaces [8]. Cleaning surfaces by faculty occurred appropriately in response to observed contamination. Between student participants, CDC guidelines were followed for kill time and appropriate product use. The room was well ventilated, extremely large and the building construction was new therefore ventilation concerns were minimized [9]. Masks were mandated to be worn for all participants throughout the simulation participation based on the CDC recommendations

"Corresponding Author: Dr. Debra Hagerty, Faculty of Nursing, Georgia Southern University, Armstrong Campus-Ashmore Hall 259, GA 30458, USA; E-mail:dhagerty@georgiasouthern.edu

Citation: Hagerty D (2021) The Use of Simulation Activities in Nursing to Increase Awareness of Patient Disabilities-Consideration of COVID-19 Practice Standards. Int J Nurs Clin Pract 8: 338. doi: https://doi.org/10.15344/2394-4978/2021/338

Copyright: (c) 2021 Hagerty. This is an open-access article distributed under the terms of the Creative Commons Attribution License, which permits unrestricted use, distribution, and reproduction in any medium, provided the original author and source are credited. 
Citation: Hagerty D (2021) The Use of Simulation Activities in Nursing to Increase Awareness of Patient Disabilities-Consideration of COVID-19 Practice Standards. Int J Nurs Clin Pract 8: 338. doi: https://doi.org/10.15344/2394-4978/2021/338

Page 2 of 3

and social distancing exceeding 6 feet between stations was established [10]. Although social distancing was required, certain simulation activities required standby supervision of the student for safety; therefore volunteers were mindful of the safety needs while maintaining a social distance of 6 feet when possible.

\section{Volunteers}

This event would not have been possible without the selfless actions and student centered faculty as well as student officers and student members of both organizations ODK, and IHI working collaboratively and interprofessionally to achieve the success of this simulation event held January 25, 2021. Faculty advisors of both IHI and ODK student chapters and student officers of both assisted in the organization of the event and facilitation and monitoring of the student experiences. Faculty volunteer support was secured to observe and guide the simulation experiences. The desire to inform and teach about disabilities and sensory impairment with aging were driving factors of this collaborative effort. The need for health professions students to be knowledgeable about the types of disabilities patients have as well as increasing their sensitivity about having a disability were motivating factors. Faculty recognized the importance of teaching students about the illnesses and comorbid conditions and resulting disabilities that occur to patients.

\section{Simulation Equipment}

The equipment for this training was obtained with a grant written for and awarded from the generosity of Georgia Health Foundation. The total award was $\$ 7100.00$ enabling a multitude of disabilities to be simulated. Each station supported an activity specific to the simulation of a disability possible throughout the lifespan. The following stations were manned by faculty and student volunteers:

1. Stroke Simulation Suit using Arm sling, leg brace, eye patch and a sucker for drooling.

2. Knee wraps for knee mobility restriction and pain

3. Shoes oversized to simulate gait difficulty

4. Tremor simulator nerve impulse activator

5. Tinnitus audible buzzing simulator

6. Leg braces mobility impairment

7. Ear phones for hearing impairment

8. Eye glasses which simulate, Glaucoma, Cataract, Retinitis Pigmentosa, Diabetic Retinopathy, Unilateral Retinal Detachment, Macular Degeneration

9. Kyphosis simulator

10. Back pain simulator

11. COPD Simulator

\section{Methods of Simulation}

Each of the stations were set up for active participation with the student participants. The following are general guidelines followed by the faculty.

1. Discuss the application of the disability to be simulated.

2. Assist in donning the simulation equipment
3. Review the experiential directions and expectations for the simulation

4. Perform the simulation

5. Debrief with the participant

6. Answer questions

7. Disinfect the equipment

Simulation experiences were enhanced and made rich by the imagination of the individual faculty. For example, donning oversized shoes to simulate a gait disturbance was supplemented with activities such as climbing up a set of stairs, walking on a throw rug and stepping over a puddle. The tremor simulation was enhanced with the participant attempting the activity of opening a pill bottle as well as attempting to sign their name and write. The tremor simulator caused an electrical impulse to be imparted in varying degrees and caused the nerves to modulate in the hand. Writing was difficult as well as dexterity was impaired. The stroke, hemiplegia simulation participant was assisted to ambulate, bend and pick up an item and sit in a wheelchair to actively experience the full effect of restricted mobility. Eye glasses were used to simulate a variety of eye diseases and disabilities and participants were asks to do normal tasks of writing, reading and mobility. Knee pain was simulated with braces that fit on the knees that were used to restrict mobility and bending to pick up items was difficult. Kyphosis, or a hunched spine was created with a brace and straps and made participants walking with a normal gait difficult and breathing was more difficult. Back pain simulator fit snug on the front and back of the chest and participants verbalized this was not comfortable to move, bend or try activities of daily living. Similarly the COPD simulator which was a brace on the chest as well as nose plugs, was modified due to COVID-19 and the nose plugs were not used during the simulation experience. The hearing tinnitus experience as well as the ear plugs used for simulation created an exciting experience for participants as they certainly could not hear normal conversation and the buzzing in the ear was extremely distracting for the participants.

\section{Summary of Debriefing}

All students praised the experiences as positive and providing deep insight into what having a disability would be like. Comments such as "this was great preparation for my future classes and nursing experiences"; "I know I will encounter a patient with this type of disability so I am glad I had this experience". "I can't imagine having this disease, I cannot see". "My grandmother walks with a cane and has weakness too; I never realized how difficult to walk this caused". During simulation the psychological safety of the students was considered, specifically as it related to any activity they were not familiar with. The tremor experience was well received yet did have some anxiety related to the degree of current stimulation. All participants said it was a valuable experience. There were no issues evidenced during this simulation experience.

\section{Conclusion}

According to Newton and Krebs [5] "patients with disabilities frequently express concern that healthcare professionals lack interest and sensitivity in meeting their needs; therefore, are less likely to seek care or follow recommendations of providers", p.234. Exposure to a multitude of disabilities through simulation was a critically important method to initiate the conversation about patients, and the possible disabilities they will encounter in clinical practice. 
Student active engagement in disability simulation adds experiential learning opportunities in clinical education where opportunities and practice sites may be limited. One in four adults in the United States, or 61 million has at least one of six disabilities: hearing, vision, cognition, mobility, self-care, or independent living [11]. To aide in the development of critical thinking skills in the health professions, simulated activities can play an important role. The variety of simulated activities used this experience can be used to replace traditional clinical experiences that are very difficult to find for students as well as augment existing opportunities [12]. Most importantly, disability awareness, education, and providing disability based simulated activities is extremely valuable as supplemental supportive education and simulation for current health professionals, including nurses in a complex healthcare environment where opportunities may be limited from time to time.

\section{Competing Interests}

The author declare that there is no competing interests regarding the publication of this article.

\section{References}

1. Watties-Daniels (2020) The impact of COVID-19 on nursing student clinical practice: A time for clinical innovation. The Maryland Nurse Journal.

2. McGuire S (2015) Teach students how to learn: Strategies you can incorporate into any course to improve student metacognition, study skills, and motivation. Stylus.

3. Tesfaye S, Berhanu K (2015) Improving students' participation in active learning methods: group discussions, presentations and demonstrations: A case of Madda Walabu university second year tourism management students of 2014. Journal of Education and Practice 6: 29-32.

4. Americans With Disabilities Act of 1990, 42 U.S.C. § 12101 et seq

5. Newton $\mathrm{RH}$, Krebs A (2020) Bridging the theory practice gap using simulation to teach care of patients with disabilities. Teaching and Learning in Nursing 15: 232-236.

6. Franklin AE, Blodgett NP (2020) Simulation in nursing education. Annual Review of Nursing Research 39: 3-31.

7. Centers for Disease Control and Prevention (2021) Cleaning and disinfecting your facility.

8. Environmental Protection Agency (2021) About list N: Disinfectants for Coronavirus (COVID-19).

9. Centers for Disease Control and Prevention (2021) Ventilation in buildings.

10. Centers for Disease Control and Prevention (2021) Use masks to stop the spread of COVID-19.

11. Okaro C, Hollis N, Cyrus A, Griffin-Blake S (2018) Prevalence of disabilities and healthcare access by disability status and type among adults-United States, 2016. MMWR Morb Mortal Wkly Rep 67: 882-887.

12. Aebersold M (2018) Simulation-based learning: No longer an novelty in undergraduate education. OJIN: The Online Journal of Issues in Nursing. 